\title{
Premature ovarian insufficiency patients with viable embryos derived from autologous oocytes through repeated oocyte retrievals could obtain reasonable cumulative pregnancy outcomes following frozen-embryo transfer
}

\author{
Xiuxian Zhu ${ }^{1,2}$, Jing $\mathrm{Ye}^{2}$, Yonglun $\mathrm{Fu}^{1,2}$ \\ ${ }^{1}$ Department of Assisted Reproductive Medicine, Shanghai First Maternity and Infant Hospital, Tongji University School of Medicine, Shanghai, \\ China; ${ }^{2}$ Department of Assisted Reproduction, Shanghai Ninth Peoples Hospital, Shanghai Jiaotong University School of Medicine, Shanghai, \\ China \\ Contributions: (I) Conception and design: X Zhu, Y Fu; (II) Administrative support: X Zhu, Y Fu; (III) Provision of study materials or patients: X Zhu, \\ J Ye; (IV) Collection and assembly of data: X Zhu, J Ye; (V) Data analysis and interpretation: X Zhu, Y Fu; (VI) Manuscript writing: All authors; (VII) \\ Final approval of manuscript: All authors. \\ Correspondence to: Yonglun Fu, MD, PhD. Department of Assisted Reproductive Medicine, Shanghai First Maternity and Infant Hospital, Tongji \\ University School of Medicine, Gaokexi Road No. 2699, Shanghai 201204, China. Email: fuyonglunivf@163.com.
}

Background: Women with premature ovarian insufficiency (POI) are often discouraged from using autologous oocytes; however, some patients have a strong desire to be genetically linked to their offspring. In the present study, we aimed to estimate cumulative pregnancy outcomes following frozen-embryo transfer (FET) in POI patients who could obtain viable embryos with their eggs during in vitro fertilization (IVF)/ intracytoplasmic sperm injection (ICSI) treatments.

Methods: In this matched-retrospective cohort study, only patients undergoing IVF/ICSI treatments with a freeze-all strategy were screened, and 103 POI patients were matched with 515 normal controls in terms of the same number of viable embryos obtained at the same age. The primary outcome was the cumulative clinical pregnancy rate (CCPR) following FET per patient.

Results: Patients with POI and normal ovarian reserve had comparable CCPRs of 62.14\% (64/103) and $65.24 \%(336 / 515)$, respectively $(\mathrm{P}=0.547)$, and no statistical difference was found in the cumulative livebirth rate (CLBR) between the study group (43.69\%) and the control group $(53.01 \%)$. Based on binary logistic regression, the CCPR and CLBR showed no association with the type of ovarian function (POI or normal ovarian reserve). The number of embryos per transfer and the sum of all viable embryos per patient were positively associated with the CCPR and CLBR. The clinical pregnancy rate (CPR) per FET cycle was $38.17 \%$ for the study group and $52.1 \%$ for the control group, while the CPRs per oocyte retrieval cycle in the 2 groups were $11.25 \%$ and $69.9 \%$, respectively, and both were statistically different $(\mathrm{P}<0.05)$. Moreover, POI patients had a lower implantation rate $(27.8 \%$ vs. $37.94 \%)$ and a higher early miscarriage rate per transfer $(26.76 \%$ vs. $15 \%)$ than patients in the control group $(\mathrm{P}<0.05)$.

Conclusions: Cumulative pregnancy outcomes following FET were reasonable for POI patients using viable embryos derived from autologous oocytes through repeated oocyte retrievals.

Keywords: Premature ovarian insufficiency (POI); autologous oocytes; repeated oocyte retrievals; frozen-thawed embryo transfer; viable embryos

Submitted Jan 30, 2020. Accepted for publication Dec 31, 2020.

doi: $10.21037 /$ atm-20-1112

View this article at: http://dx.doi.org/10.21037/atm-20-1112

(c) Annals of Translational Medicine. All rights reserved. 


\section{Introduction}

Premature ovarian insufficiency (POI) represents a continuum of ovarian dysfunction before the age of 40 , and approximately one in 100 women under the age of 40 will experience POI. Because the probability of spontaneous pregnancy without medical intervention is low in infertile women with POI, many patients undertake in vitro fertilization (IVF)/intracytoplasmic sperm injection (ICSI) treatments for infertility after a diagnosis of POI (1-3). However, women with POI are often discouraged from using autologous oocytes in IVF/ICSI cycles because of the high cancellation rate during ovarian stimulation, the small number of available oocytes, and poor oocyte potential quality, and the large economic burden (1-3).

The American Society for Reproductive Medicine (ASRM) considers treatment "futile" if the live-birth rate (LBR) per cycle of treatment is less than $1 \%$ and a "very poor prognosis" when the LBR per cycle of treatment is between 1 and $5 \%$ (4). A few studies related to the pregnancy outcomes of patients with a "very poor prognosis" have been published. In an analysis of 8,019 IVF cycles with autologous oocytes, Scott et al. identified a baseline threshold of follicle stimulating hormone (FSH) at $18 \mathrm{IU} / \mathrm{L}$, beyond which no women conceived (5). Martin et al. found that no pregnancies occurred in 53 cycles with basal FSH levels only $\geq 20$ IU/L, while the clinical pregnancy rate (CPR) per cycle was $16.5 \%$ in 1,750 women whose basal FSH levels were always <20 IU/L (6). Also, the CPR per cycle was reported to be $5.6 \%$ in 54 cycles where only 1 previous FSH determination was $\geq 20$ IU/L, with a current-day $3 \mathrm{FSH}<20 \mathrm{IU} / \mathrm{L}$, and $0 \%$ in 11 cycles in which basal FSH was $<20$ IU/L during the treatment cycle but with a basal FSH $\geq 20$ IU/L 2 or more times (6). In another large study performed in 2,057 patients with 3,401 IVF/ ICSI cycles, investigators reported an LBR per cycle of $3 \%$ in 33 patients with a basic FSH > 20 IU/L, an LBR of $16.7 \%$ for women younger than 38 years, $0 \%$ for women $>38$ years, and a cumulative live-birth rate (CLBR) per patient of $19.2 \%$ after 3 IVF cycles (7). These studies support the notion that it is difficult for patients with a poor prognosis to conceive with autologous oocytes. Thus, oocyte donation is recommended to increase the chances of pregnancy in this population.

However, oocytes' usage from donors is still limited or even prohibited because of cultural, religious, or ethical issues in some countries. Additionally, some POI patients have a strong desire to be genetically linked to their offspring. Many POI patients come to our clinic insisting on pursuing IVF with their eggs, despite the slim chances of success. In our department, repeated oocyte retrievals and frozen-embryo transfer (FET) are recommended as the routine methods for women with POI. No articles have been published reporting the cumulative pregnancy rate of FET in POI patients using autologous oocytes. Herein, we collected POI patients' data who could obtain viable embryos through repeated oocyte retrievals during IVF/ ICSI treatments with a freeze-all strategy and compared the cumulative pregnancy outcomes following FET in POI patients to patients with normal ovarian reserve. We aimed to demonstrate whether POI patients with viable embryos derived from autologous oocytes can achieve reasonable cumulative pregnancy outcomes.

\section{Methods}

\section{Study setting and ethics approval}

Our study was approved by the Shanghai Ninth People's Hospital's Institutional Review Board (IRB) (No: 2015-53). It was conducted according to the Declaration of Helsinki (as revised in 2013). The data in our study were obtained from the electronic database that included all medical records of patients who underwent IVF/ICSI treatments at the Department of Assisted Reproduction of the Ninth People's Hospital affiliated with Shanghai JiaoTong University School of Medicine between January 2012 and June 2019. In our unit, informed consent relevant to infertility treatments and data usage for research purposes on the premise of anonymous identities and confidential medical records was obtained from all the patients.

\section{Study design and population}

This was a matched-retrospective cohort study in which we compared the cumulative pregnancy outcomes following FET between POI patients and patients with normal ovarian reserve. POI was defined as oligo/amenorrhea for at least 4 months, and an elevated FSH level >25 IU/L on 2 occasions $>4$ weeks apart in women younger than 40 years of age, according to the European Society of Human Reproduction and Embryology (ESHRE) guidelines regarding POI (1). Analysis of our study group only included patients with POI who had completed at least 1 FET cycle.

All patients included in our study underwent IVF/ 
ICSI with a freeze-all strategy. Ovarian stimulation cycles producing at least 1 dominant follicle with a diameter larger than $12 \mathrm{~mm}$ were selected, and ovarian stimulation cycles initiated without dominant follicles were excluded from our analyses. Viable embryos were obtained through repeated oocyte retrievals in POI patients, while patients in the control group obtained viable embryos during their first oocyte pick-up (OPU) cycle at our center. We excluded women who failed to become pregnant but still had frozen embryos remaining and women with core data missing in the electronic medical records or lost to follow-up. Delivery of multiple offspring was considered as 1 live birth in our study.

We selected normal ovulatory women undergoing their first IVF/ICSI cycle at our department as potential controls. The conditions for selecting matched controls were women with the same number of viable embryos at the same age. For repeated oocyte retrievals in POI patients, the number of viable embryos referred to the sum of viable embryos obtained from all oocyte retrieval cycles in 1 POI patient. In contrast, in normally ovulating patients, it was defined as the number of viable embryos derived from 1 oocyte retrieval cycle. Since oocyte retrievals were completed over more than 1 year for POI patients, the matched age in the control group was considered as the age of the first viable embryo obtained for POI patients in our department. The procedure for performing the 1:5 matching was as follows: First, we selected the potential control list from the control group; second, we assigned a random number for each patient using the random number generator function in Microsoft Excel; third, we placed the numbers in numerical order (from lowest to highest) according to the allocated number, and the first 5 patients were then made controls. If there was more than 1 POI patient who shared the same matched condition, the number of controls was multiplied by the number of cases in the study group (i.e., the first 15 controls were chosen as the controls if there were 3 POI patients with the same number of viable embryos at the same embryonic age). After selecting all the participants, the corresponding FET cycles were screened for subsequent analysis. The pregnancy outcomes were followed up by June 30, 2019.

In POI patients, ovarian stimulation and oocyte retrieval was performed randomly, during menstruation, in the early-late follicular phase, or the luteal phase. Flexible ovarian stimulation protocols have been adopted (8-10), including their natural cycle and the use of either mild stimulation using clomiphene citrate (CC) or letrozole, gonadotropin-releasing hormone (GnRH) agonists, $\mathrm{GnRH}$ antagonists, hormone-replacement therapy (HRT), double ovarian stimulation, or progesterone related protocols. The medications used were adjusted according to the results of transvaginal ultrasonographic examinations and serum hormone measurements. Medical records of the ovarian stimulation cycles initiated without large follicles were incomplete, and we therefore excluded them from the electronic database and only included ovarian stimulation cycles with dominant follicles larger than $12 \mathrm{~mm}$ in diameter on the trigger day. An ovarian stimulation cycle without preovulation was calculated as 1 OPU cycle.

In the control group, the method for endometrial preparation in FET cycles was based on our clinic's routine procedures, including natural cycle, mild stimulation, and HRT, which have been elaborated in our previous studies (8-10). For POI patients, HRT was generally the preferred method. Briefly, 2 red Femoston (Abbott Healthcare Products B.V.) tablets containing $2 \mathrm{mg}$ of estradiol per tablet were administered twice a day from the beginning of the FET cycle onwards. When the endometrial thickness reached $\geq 7 \mathrm{~mm}$, the red tablets were replaced by 2 yellow Femoston tablets (composed of $2 \mathrm{mg}$ of estradiol and $20 \mathrm{mg}$ of dydrogesterone), co-administered with $400 \mathrm{mg}$ of natural micronized progesterone (Utrogestan, Laboratories Besins International, Paris, France). Cleavage-stage embryo transfer was scheduled 3 days after progesterone administration, while FET was performed on the fifth day for blastocysts. Once pregnancy was achieved, progesterone was taken until 10 weeks of gestation.

\section{Outcome measures}

The primary outcome of our study was the cumulative clinical pregnancy rate (CCPR) per patient. CLBR per patient, CPR per FET cycle, LBR per FET cycle, CPR per OPU cycle, LBR per OPU cycle, implantation rate, miscarriage rate, and biochemical pregnancy rate were the secondary outcome measures. Biochemical pregnancy was defined as a serum level of human chorionic gonadotropin $>5 \mathrm{mIU} / \mathrm{mL}$. The presence of a gestational sac with or without fetal heart activity in the uterine cavity, ectopic pregnancy, or heterotopic pregnancy was defined as a clinical pregnancy. Spontaneous pregnancy termination before the gestational age of 12 weeks was considered as an early miscarriage. Live birth was defined as a live-born baby at 24 gestational weeks or later. The implantation rate was calculated as the number of observed gestational sacs to the 


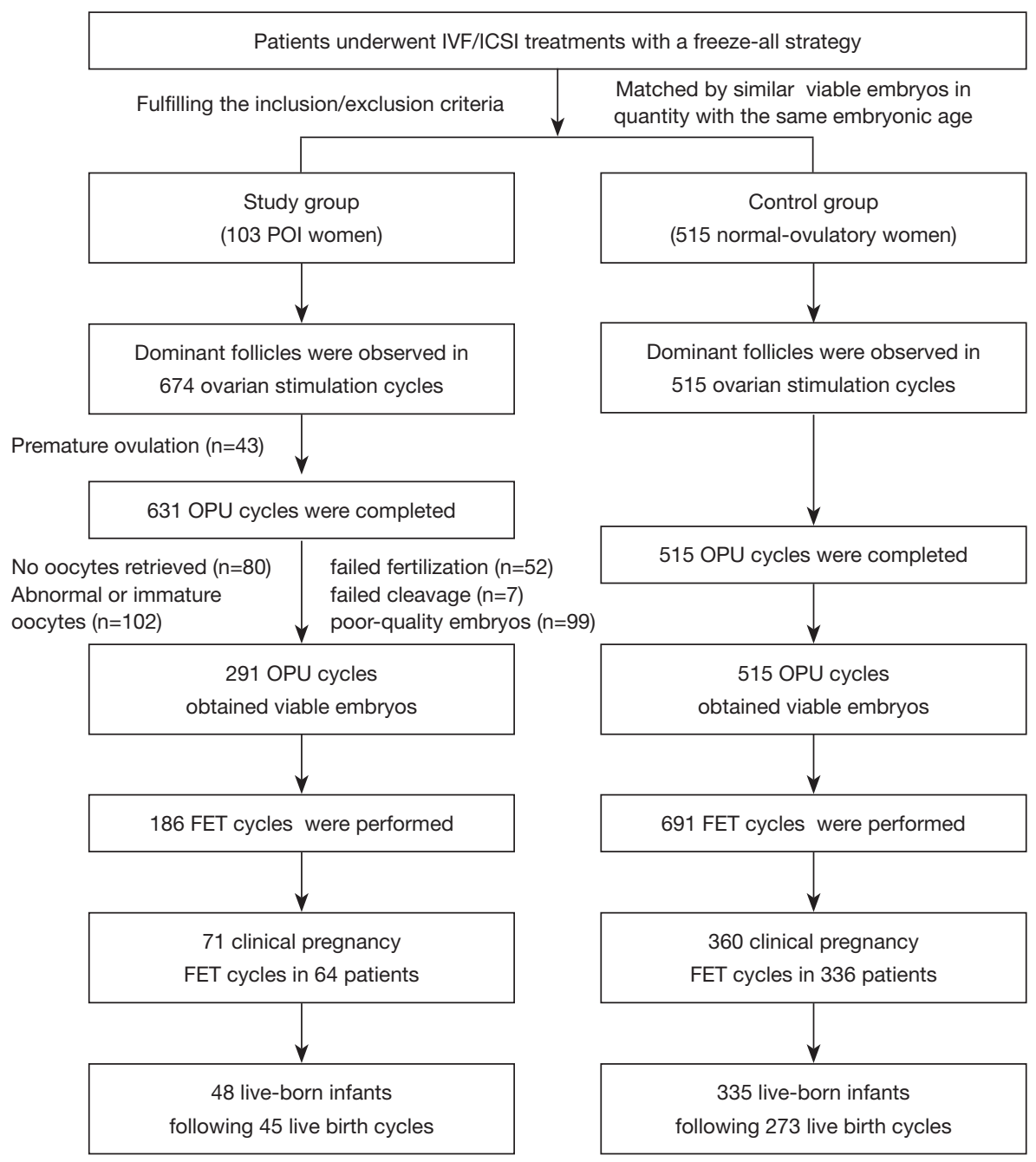

Figure 1 Flow chart of the study. IVF, in vitro fertilization; ICSI, intracytoplasmic sperm injection; FET, frozen embryo transfer; OPU, oocyte pick-up.

number of transferred embryos.

\section{Statistical analysis}

All analyses were performed using the Statistical Package for the Social Sciences for Windows, version 24.0 (SPSS, Chicago, IL, USA). Data were presented as medians and interquartile range for non-normally distributed data means \pm standard deviation (SD) for normally distributed data, and percentages for categorical data. Student's $t$-tests, MannWhitney $\mathrm{U}$ tests, and Chi-square tests were performed for statistical analyses. We also performed a binary logistic regression to quantify the effects of risk factors on the CCPR and CLBR. The age of the embryo, body mass index
(BMI), group (control vs. study), the number of transferred embryos per attempt, and the number of all viable embryos per patient were considered as the independent variables in the regression equation, using the enter method. We presented the effects of risk factors as an adjusted odds ratio $(\mathrm{OR})$ and $95 \%$ confidence interval (CI). A $\mathrm{P}$ value less than 0.05 was considered statistically significant.

\section{Results}

The flow chart of the study is illustrated in Figure 1. Data from a total of 103 women with POI were eligible to be analyzed in the study group. There were 674 ovarian stimulation cycles scheduled for OPU in the study group. 

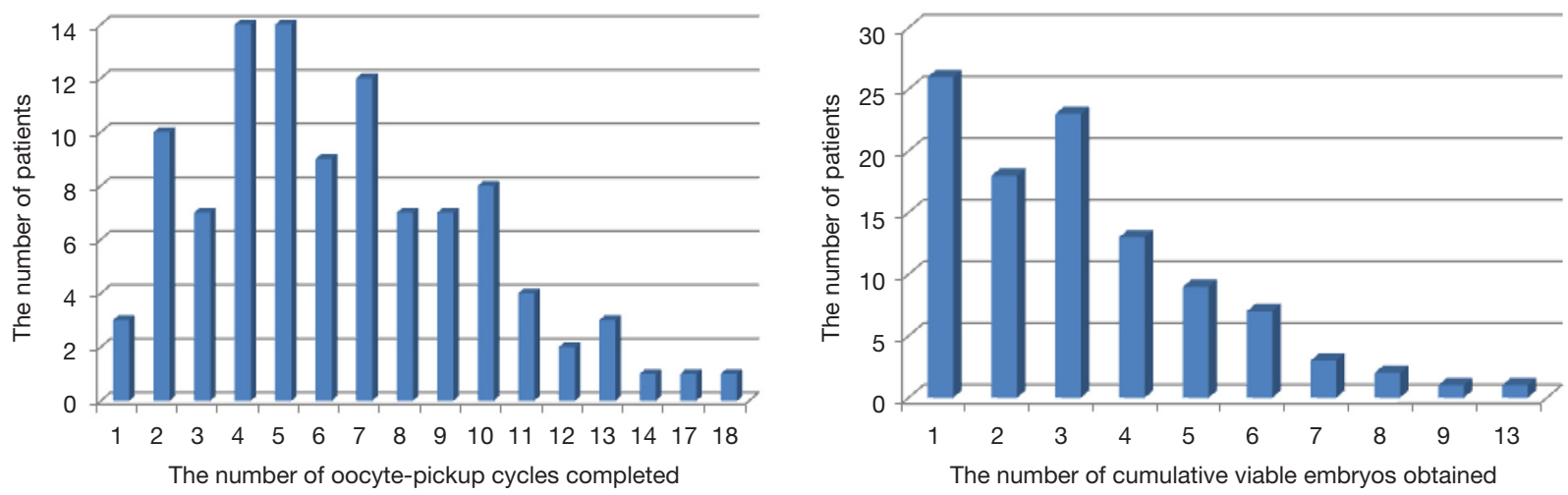

Figure 2 The distribution of patients in terms of the number of oocyte pick-up cycles and viable embryos.

Of these cycles, 43 cycles $(7.4 \%)$ were in women who experienced premature ovulation, 80 cycles $(17.8 \%)$ failed to produce retrievable oocytes, 102 cycles had abnormal or immature oocytes, 52 cycles resulted in failed fertilization, 7 cycles failed to produce cleavage-stage embryos, and 99 cycles produced poor-quality embryos. Finally, 329 viable embryos were obtained through 291 OPU cycles in the 103 POI patients. The 103 POI patients in the study group were then compared with 515 matched controls (5 controls per patient) according to our inclusion/exclusion criteria. During the study period, POI patients completed 186 FET cycles, while 515 normal ovulatory patients completed 691 FET cycles.

Figure 2 depicts the distribution of patients in terms of the number of OPU cycles and viable embryos. In the study group, 1 patient underwent a maximum of 18 OPU cycles, and the maximal number of viable embryos was 13 in 1 patient.

The pregnancy and live-birth outcomes following FET for all patients are shown in Table 1. A total of 1,428 embryos were thawed, and the rate of viable frozen-thawed embryos was $97.41 \%(1,391 / 1,428)$. In the study group, single embryos were transferred in 96 FET cycles, and multiple embryos were transferred in 90 FET cycles. In the control group, single embryos were transferred in 238 FET cycles, and multiple embryos were transferred in 452 FET cycles. CPR per FET cycle in the study group was lower than that in the control group (38.17\% vs. $52.1 \%)$, and CPR per OPU cycle in the study group was also lower than that in the control group $(11.25 \%$ vs. $69.9 \%)$, with statistically significant differences $(\mathrm{P}<0.05)$. Moreover, significant differences were also found in the rates of biochemical pregnancy, multiple pregnancies, implantation, early miscarriage, and live-births per transfer $(\mathrm{P}<0.05)$. In contrast, the CCPR per patient was $62.14 \%(64 / 103)$ in the study group and $65.24 \%(336 / 515)$ in the control group, though the difference was not significant $(\mathrm{P}>0.05)$, and the CLBR per patient was also similar between the study group (43.69\%) and the control group (53.01\%). A total of 312 patients completed deliveries during our follow-up, including 42 cycles with term delivery in the study group and 238 cycles with term delivery in the control group $(\mathrm{P}>0.05)$. The birth weights of the single newborns were comparable between the two groups, while the birth weights of twin newborns in the study group were lower than in the control group $(\mathrm{P}=0.046)$.

Table 2 presents the results of unadjusted and adjusted analyses for possible factors affecting the CCPR and CLBR in all patients. The results show a statistically significant increase in CCPR and CLBR with the increase in the number of transferred embryos per transfer and the number of all viable embryos per patient in both the unadjusted and adjusted models. The increase in viable embryos' age was negatively related to CLBR (OR, 0.944; 95\% CI: $0.904-$ 0.986; $\mathrm{P}=0.01$ for the unadjusted model, and $\mathrm{OR}, 0.944$; 95\% CI: 0.903-0.986; $\mathrm{P}=0.009$ for the adjusted model). Based on the binary logistic regression, the CCPR and CLBR showed no association with the ovarian function type (i.e., POI or normal ovarian reserve).

Figure 3 presents the cumulative pregnancy outcomes according to the patients' age. For women aged $\leq 34$, $35-37$, or $\geq 38$ in the study group, the CCPRs were $65 \%$, $59.26 \%$, and $59.25 \%$, respectively, and the CLBRs were $46.67 \%, 40.74 \%$, and $37.5 \%$, respectively, which shows a diminished tendency with increasing age (this was not statistically significant; trend analysis, $\mathrm{P}=0.468$ for CCPR 


\section{Page 6 of 11}

Zhu et al. Reasonable cumulative pregnancy outcomes in POI patients

Table 1 Pregnancy and live-birth outcomes of frozen-thawed embryo transfers in patients with premature ovarian insufficiency and matched normal-ovulatory controls

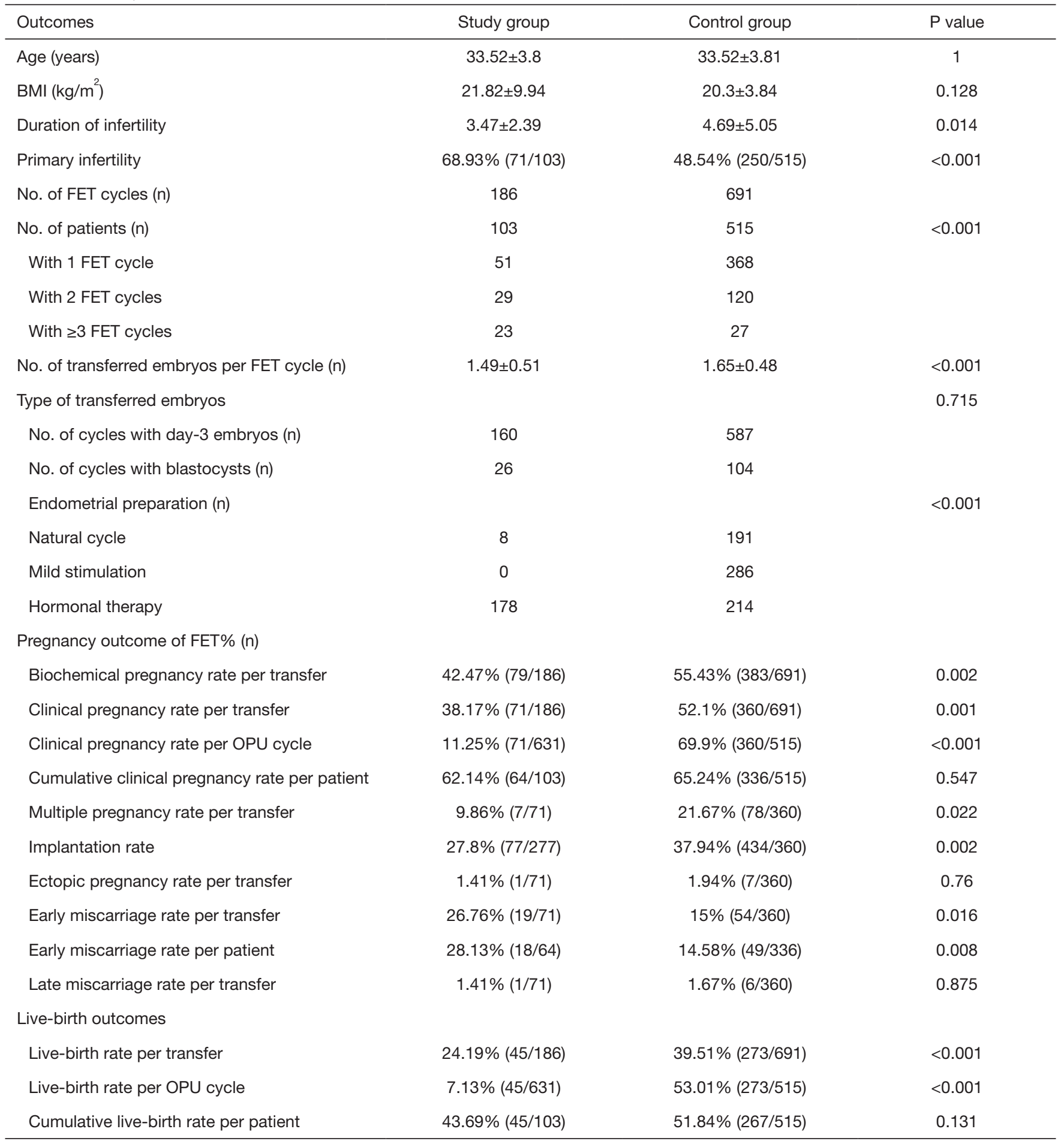

Table 1 (continued) 
Table 1 (continued)

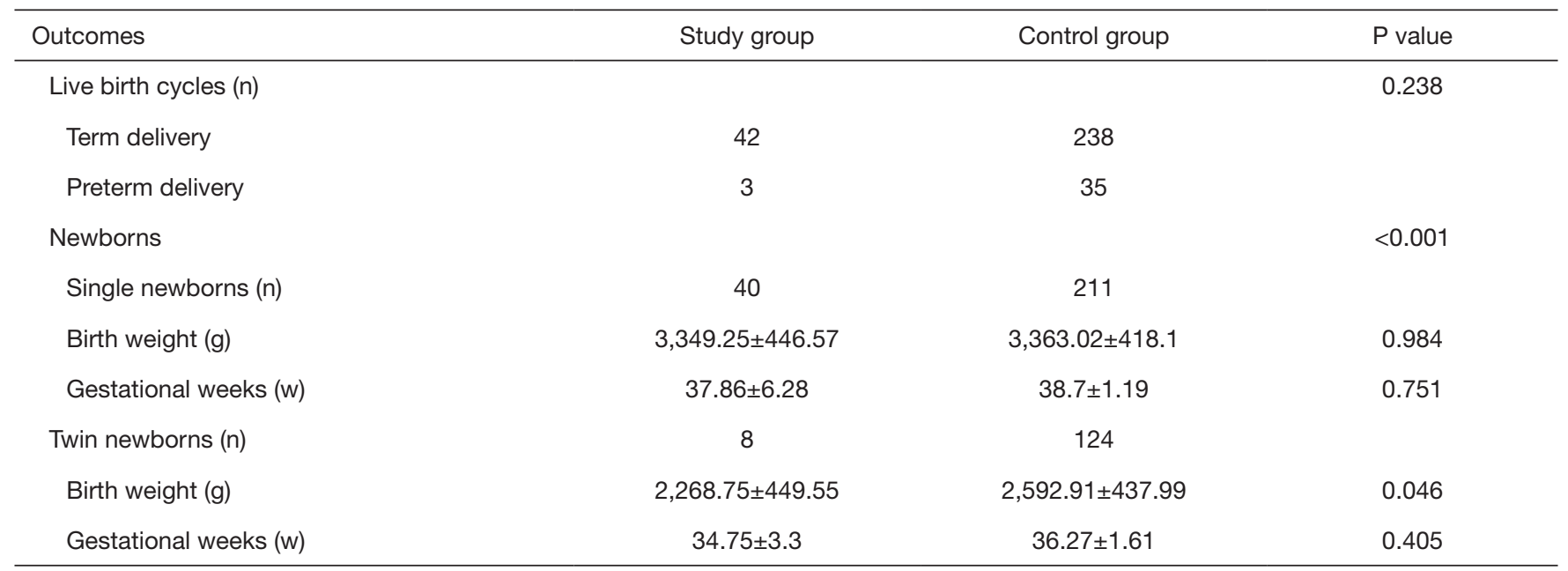

For categorical variables\%, $(n)$ is presented; for continuous variables, mean (SD) is presented. For comparisons between groups, Pearson's Chi-square test or Fisher's exact test was used for categorical variables and the Mann-Whitney U test was used for continuous variables. FET, frozen embryo transfer; OPU, oocyte-pickup; BMI, body mass index.

and $\mathrm{P}=0.458$ for CLBR). No statistical significance was found between the two groups regarding the CCPR and CLBR for different ages. However, the control group's cumulative early miscarriage rate significantly increased with increments in age (trend analysis, $\mathrm{P}=0.004$ ), although this trend was not found in the study group (trend analysis, $\mathrm{P}=0.83$ ). Also, the cumulative early miscarriage rate was higher in the study group relative to the control group among patients under the age of 34, while it was similar in the two groups in patients older than 35 .

\section{Discussion}

During IVF/ICSI treatments, one of the primary challenges is managing infertile women with POI. Many reproductive centers refuse to treat POI patients because the chance of success is thought to be low using IVF with autologous oocytes. To the best of our knowledge, our study is the first study reporting the cumulative pregnancy outcomes in POI patients undergoing IVF/ICSI with autologous oocytes using a freeze-all strategy. Our data showed that the CCPR was comparable between POI patients and matched normal controls.

In our study, the CPRs per FET cycle and per OPU cycle in matched normal controls were 1.4 times and 6-fold greater than those in POI patients, respectively. However, the CCPR per patient was comparable between the 2 groups. Although the LBRs per OPU cycle and
FET cycle for POI patients in our study were $7.13 \%$ and $24.19 \%$, respectively, the CLBR per patient reached $43.69 \%$, which was better than previously reported and far exceeded the ASRM definitions of "futile treatment" and "very poor prognosis". From our perspective, the increase in CCPR and CLBR may be attributed to the method of repeated oocyte retrievals and FET. With the development of cryopreservation techniques, FET has been adopted by an increasing number of clinics. Chen et al. recently demonstrated that FET resulted in a lower risk of ovarian hyperstimulation syndrome and was associated with a higher rate of live births among infertile women with polycystic ovary syndrome $(11,12)$. The freezeall strategy created flexibility in ovarian stimulation without the endometrium's constraints due to medications or abnormal blood hormone concentrations. Whenever there were antral follicles, ovarian stimulation could commence independent of the menstrual cycle. POI patients could accumulate more embryos through repeated oocyte retrievals and embryo cryopreservation, which may remedy the quantitative reduction in the ovarian reserve. Thus, we believe the method of repeated oocyte retrievals and FET may be beneficial for POI patients' treatment.

Some researchers have claimed that more attention should be paid to infertile women's age rather than FSH levels. Kushnir et al. reported an LBR of $8.6 \%$ per patient and $6 \%$ per initiated IVF cycle in patients with a maximal baseline FSH $>20$ IU/L. The LBR was also 
Table 2 Binary logistic regression regarding cumulative clinical pregnancy and live births following frozen-thawed embryo transfers

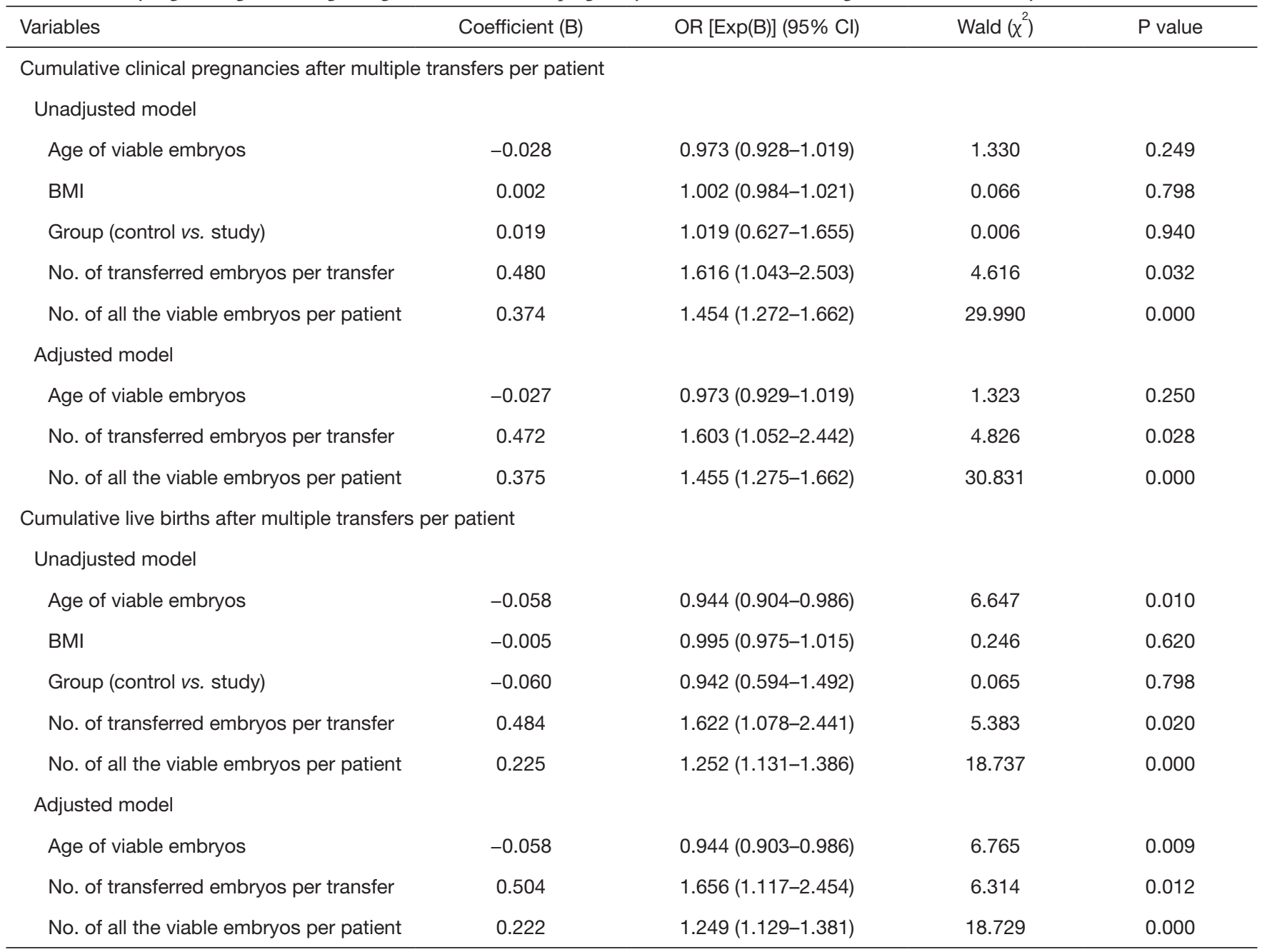

$\mathrm{OR}$, odds ratio; $\mathrm{Cl}$, confidence interval. $\mathrm{P}$ value shows significance of input into the logistic regression model; $\mathrm{P}$ values in bold indicate statistical significance. FET, frozen embryo transfer; BMI, body mass index.
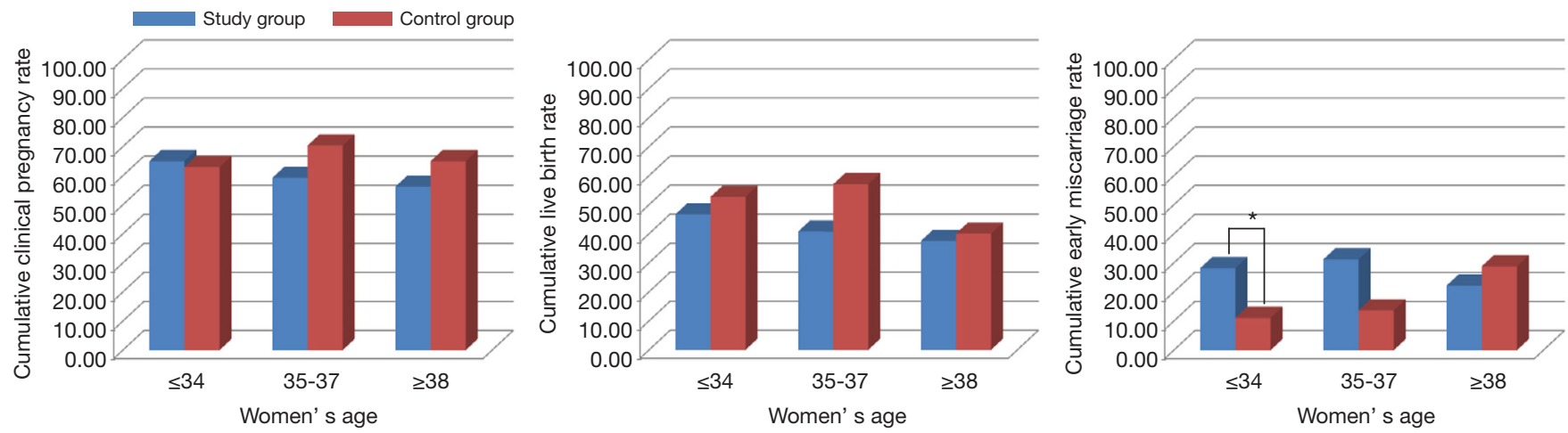

Figure 3 Age-specific cumulative pregnancy outcomes in the 2 groups. ${ }^{*} \mathrm{P}<0.05$ at the specific time-point. 
negatively associated with maternal age, with the highest LBR of $17.2 \%$ in younger women (under 35 years of age) and the lowest LBR of $1.9 \%$ among women older than 42 years of age (3). Based on a series of analyses (13-15), Kushnir et al. concluded that elevated FSH levels should not be considered as the exclusive factor for predicting the chance for pregnancy during IVF with autologous oocytes. A successful live birth in this patient population was positively related to a younger age, the number of oocytes, and transferable embryos (13-15). Morin et al. observed equivalent blastocyst formation rates, aneuploidy rates, and LBRs per euploid embryo transfer between young poor-responders and age-matched normal controls (16), suggesting that the lower LBR in young women with evidence of accelerated follicular depletion was related to a quantitative reduction in the number of oocytes retrieved but not in attenuation in oocyte quality. In our study, binary logistic regression showed a statistically significant influence of age and the number of all viable embryos on the CLBR. However, the CCPR per patient was not associated with age, which suggested that the accumulation of viable embryos may compensate for a woman's age through repeated oocyte retrievals.

High cancellation rates are a difficult issue during repeated oocyte retrieval cycles. In an analysis of 2,386 consecutive cycles of IVF/ICSI in women 40 years and older, Serour et al. detected a linear increase in the cancellation rate with age increments and calculated a cancellation rate of $16 \%$ per initiated cycle (17). This is similar to the $16.6 \%$ in 1,217 cycles reported by Tsafrir et al. (18) and the $19.9 \%$ in 2,705 cycles reported by Klipstein et al. (19). In our study, 6.38\% (43/674) of ovarian stimulation cycles manifested premature ovulation, and $12.68 \%$ (80/631) of ovarian stimulation cycles failed to produce oocytes. Gleicher et al. reported that the probability of retrieving at least 1 oocyte in infertile women who had FSH levels between 20 and 40 IU/L ranged from $90 \%$ to $76 \%$ for those under 35 and ranged from $75 \%$ to $53 \%$ for those $\geq 43$ years of age (15). Only POI patients who obtained viable embryos and completed at least 1 FET cycle were selected and analyzed in our study.

Moreover, we only included ovarian stimulation cycles with dominant follicles. The majority of our POI patients lived a distance away from our hospital, such that some patients chose to receive medications and undergo examinations at a local hospital and then travel to our hospital for OPU. If ovarian stimulation was initiated without dominant follicles, cycles were canceled under the guidance of a doctor. As a result, medical records of the ovarian stimulation cycles initiated without large follicles were incomplete and were not included in the electronic database. Thus, we may have underestimated the cancellation rate in our study, one of our study limitations.

Another point that required our attention was whether the likelihood of early miscarriage increased in women with low ovarian reserve. In the Kushnir study (3), the rate of early miscarriage was $27 \%$ (10/37), which was an expected rate given the patients' mean age. Therefore, the authors suggested no association between the ovarian reserve and the risk of miscarriage. Conversely, some investigators have suggested that the parameters related to ovarian reserve were related to chromosomal abnormalities and the potential of miscarriage. Trout et al. observed that the day 3 serum FSH level was significantly higher in women with unexplained recurrent pregnancy loss relative to women with a known cause of miscarriage (20). Atasever et al. found that women with FSH $\geq 11 \mathrm{U} / \mathrm{L}$ levels had a higher incidence of recurrent miscarriage compared with age-matched controls (21). Our data showed that 18 $(28.13 \%)$ women experienced a spontaneous abortion out of the 64 women who achieved clinical pregnancies in the study group, which was more than that in the matched controls. Some researchers have attributed the increased risk of pregnancy wastage to alterations in immune and inflammatory markers in patients with low ovarian reserve. Considering the small sample size, the autoimmune aetiologies of POI, and the absence of cytogenetic testing of miscarriages in the present study, we suggest that our data needs to be viewed with some caution, and further study is certainly warranted.

\section{Conclusions}

Our study demonstrated that the cumulative pregnancy outcomes following FET were reasonable for POI patients who could produce viable embryos after repeated oocyte retrievals. Our data may provide valuable information for POI patients who insist on using their oocytes, despite the lower implantation rate and higher early miscarriage rate. Nevertheless, our data should be viewed with caution since only patients from whom viable embryos could be obtained were included, which might have overestimated the success rate for POI patients. Additional prospective studies that include all data for each initiated ovarian stimulation cycle in POI patients are still needed to elucidate the probability of a live birth per initiated cycle. 


\section{Acknowledgments}

The authors would like to express our sincere gratitude to the patients and the Department of Assisted Reproduction staff at Shanghai Ninth People's Hospital.

Funding: This study was funded by grants from the Science and Technology Commission of Shanghai Municipality (grant numbers 18411963800) and the Shanghai First Maternity and Infant Hospital affiliated to Tongji University School of Medicine (grant number 2020RC02).

\section{Footnote}

Data Sharing Statement: Available at http://dx.doi. org/10.21037/atm-20-1112

Conflicts of Interest: All authors have completed the ICMJE uniform disclosure form (available at http://dx.doi. org/10.21037/atm-20-1112). The authors have no conflicts of interest to declare.

Ethical Statement: The authors are accountable for all aspects of the work in ensuring that questions related to the accuracy or integrity of any part of the work are appropriately investigated and resolved. Our study was approved by the Shanghai Ninth People's Hospital's Institutional Review Board (IRB) (No: 2015-53). It was conducted according to the Declaration of Helsinki (as revised in 2013). informed consent relevant to infertility treatments and data usage for research purposes on the premise of anonymous identities and confidential medical records was obtained from all the patients.

Open Access Statement: This is an Open Access article distributed in accordance with the Creative Commons Attribution-NonCommercial-NoDerivs 4.0 International License (CC BY-NC-ND 4.0), which permits the noncommercial replication and distribution of the article with the strict proviso that no changes or edits are made and the original work is properly cited (including links to both the formal publication through the relevant DOI and the license). See: https://creativecommons.org/licenses/by-nc-nd/4.0/.

\section{References}

1. European Society for Human Reproduction and Embryology (ESHRE) Guideline Group on POI, Webber L, Davies M, et al. ESHRE Guideline: management of women with premature ovarian insufficiency. Hum Reprod 2016;31:926-37.

2. Pastore LM, Christianson MS, Stelling J, et al. Reproductive ovarian testing and the alphabet soup of diagnoses: DOR, POI, POF, POR, and FOR. J Assist Reprod Genet 2018;35:17-23.

3. Kushnir VA, Safdie M, Darmon SK, et al. Age-Specific IVF outcomes in infertile women with baseline FSH Levels $\geq 20 \mathrm{mIU} / \mathrm{mL}$. Reprod Sci 2018;25:893-8.

4. Ethics Committee of the American Society for Reproductive Medicine 2012. Fertility treatment when the prognosis is very poor or futile: a committee opinion. Fertil Steril 2012;98:e6-9.

5. Scott RT, Elkind-Hirsch KE, Styne-Gross A, et al. The predictive value for in vitro fertility delivery rates is greatly impacted by the method used to select the threshold between normal and elevated basal follicle-stimulating hormone. Fertil Steril 2008;89:868-78.

6. Martin JS, Nisker JA, Tummon IS, et al. Future in vitro fertilization pregnancy potential of women with variably elevated day 3 follicle-stimulating hormone levels. Fertil Steril 1996;65:1238-40.

7. Abdalla H, Thum MY. An elevated basal FSH reflects a quantitative rather than qualitative decline of the ovarian reserve. Hum Reprod 2004;19:893-8.

8. Zhu X, Zhang X, Fu Y. Utrogestan as an effective oral alternative for preventing premature luteinizing hormone surges in women undergoing controlled ovarian hyperstimulation for in vitro fertilization. Medicine (Baltimore) 2015;94:e909.

9. Zhu X, Ye H, Fu Y. Duphaston and human menopausal gonadotropin protocol in normally ovulatory women undergoing controlled ovarian hyperstimulation during in vitro fertilization/intracytoplasmic sperm injection treatments in combination with embryo cryopreservation. Fertil Steril 2017;108:505-12.

10. Zhu X, Fu Y. Evaluation of ovarian stimulation initiated from the late follicular phase using human menopausal gonadotropin alone in normal-ovulatory women for treatment of infertility: a retrospective cohort study. Front Endocrinol (Lausanne) 2019;10:448.

11. Shi Y, Sun Y, Hao C, et al. Transfer of fresh versus frozen embryos in ovulatory women. N Engl J Med 2018;378:126-36.

12. Chen ZJ, Shi Y, Sun Y, et al. Fresh versus frozen embryos for infertility in the polycystic ovary syndrome. N Engl J Med 2016;375:523-33.

13. Gleicher N, Vega MV, Darmon SK, et al. Live-birth rates 
in very poor prognosis patients, who are defined as poor responders under the Bologna criteria, with nonelective single embryo, two embryo, and three or more embryos transferred. Fertil Steril 2015;104:1435-41.

14. Vega MG, Gleicher N, Darmon SK, et al. IVF outcomes in average-and poor-prognosis infertile women according to the number of embryos transferred. Reprod Biomed Online 2016;33:370-5.

15. Gleicher N, Darmon SK, Kushnir VA, et al. How FSH and $\mathrm{AMH}$ reflect probabilities of oocyte numbers in poor prognosis patients with small oocyte yields. Endocrine 2016;54:476-83.

16. Morin SJ, Patounakis G, Juneau CR, et al. Diminished ovarian reserve and poor response to stimulation in patients <38 years old: a quantitative but not qualitative reduction in performance. Hum Reprod 2018;33:1489-98.

17. Serour G, Mansour R, Serour A, et al. Analysis of 2,386 consecutive cycles of in vitro fertilization or

Cite this article as: Zhu X, Ye J, Fu Y. Premature ovarian insufficiency patients with viable embryos derived from autologous oocytes through repeated oocyte retrievals could obtain reasonable cumulative pregnancy outcomes following frozen-embryo transfer. Ann Transl Med 2021;9(7):539. doi: 10.21037/atm-20-1112 intracytoplasmic sperm injection using autologous oocytes in women aged 40 years and above. Fertil Steril 2010;94:1707-12.

18. Tsafrir A, Simon A, Revel A, et al. Retrospective analysis of 1217 IVF cycles in women aged 40 years and older. Reprod Biomed Online 2007;14:348-55.

19. Klipstein S, Regan M, Ryley DA, et al. One last chance for pregnancy: a review of 2,705 in vitro fertilization cyclesinitiated in women age 40 years and above. Fertil Steril 2005;84:435-45.

20. Trout SW, Seifer DB. Do women with unexplained recurrent pregnancy loss have higher day 3 serum FSH and estradiol values? Fertil Steril 2000;74:335-7.

21. Atasever M, Soyman Z, Demirel E, et al. Diminished ovarian reserve: is it a neglected cause in the assessment of recurrent miscarriage? A cohort study. Fertil Steril 2016;105:1236-40. 